\title{
Exciton in a quantum wire in the presence of parallel and perpendicular magnetic fields
}

\author{
Y. Sidor,* B. Partoens, and F. M. Peeters ${ }^{\dagger}$ \\ Departement Fysica, Universiteit Antwerpen (Campus Drie Eiken), Universiteitsplein 1, B-2610 Antwerpen, Belgium
}

(Received 15 November 2004; published 26 April 2005)

\begin{abstract}
The exciton properties of self-assembled rectangular and V-shaped quantum wires are investigated theoretically in the presence of a magnetic field. The calculations are done in the single band effective mass approximation. We study the diamagnetic shift, the influence of the electron-hole Coulomb interaction, as well as the electronic properties and the photoluminescence peak energies for magnetic fields applied along and perpendicular to the wire. The results are compared with available magneto-photoluminescence experimental measurements.
\end{abstract}

DOI: 10.1103/PhysRevB.71.165323

PACS number(s): 73.21.Hb, 71.35.Ji, 75.75.+a

\section{INTRODUCTION}

The investigation of low-dimensional structures, such as quantum wires (QWRs) and quantum dots (QD), have attracted much attention for potential application in highperformance devices since they are theoretically predicted to offer superior optical and electrical characteristics. ${ }^{1,2}$ A few main techniques exist to fabricate QWRs, such as electron beam lithography and etching, growth on nonplanar substrates, selective growth on masked substrate, molecular beam epitaxy, and cleaved-edge overgrowth method. Different shapes for QWRs using the aforementioned methods have been realized; for example, the T-shaped QWRs ${ }^{3-5}$ and the V-shaped QWRs (Refs. 6-10) have been studied theoretically and investigated experimentally. Early work for rectangular-shaped QWRs by Degani et al. ${ }^{11}$ and later several other studies used model calculations to describe the properties of excitons confined to unstrained QWRs. ${ }^{12-14} \mathrm{Ap}-$ proaches, which show the role of electron-hole Coulomb interaction together with realistic profiles of the confining potential (see for example Refs. 12 and 15) have also been presented. On the other hand, valence-band mixing effects in the T-shaped and the V-shaped QWRs was studied by Goldoni et al. ${ }^{16}$ using four-band $\mathbf{k} \cdot \mathbf{p}$ theory, and by Stier $e t$ al. ${ }^{17}$ using eight-band $\mathbf{k} \cdot \mathbf{p}$ theory, which was later applied to rectangular-shaped QWRs. ${ }^{18}$ In all the latter cases the Coulomb interaction was neglected and the calculation was done for zero magnetic field. There has been much theoretical interest in the study of the magnetic properties of QWRs. Kyrychenko et al. ${ }^{19}$ performed calculations of the valence band states in diluted semiconductor QWR structures. They showed that it is possible to control the relative contribution of the light- and the heavy- hole components of the total wave function of the holes by changing an external magnetic field. Bryant et al. ${ }^{20}$ calculated the diamagnetic shift in T-shaped QWRs with perturbation theory; lately, Madureira et $a l^{21}$ have investigated the magnetoexciton states and optical-absorption spectrum in QWRs. They found an enhancement of the exciton binding energy with increasing magnetic field, as was expected from experimental data.

Recently, considerable effort was devoted to the achievement of self-assembled QWRs, which can be formed under certain growth conditions by solid source molecular beam epitaxy. In this case the wires are formed by the Stranski-
Krastanow growth mode, in which the materials that are deposited on top of each other have a substantially different lattice parameter. Spontaneous formation of self-assembled InAs QWRs on InP (001) substrate, having 3.2\% lattice mismatch, was recently demonstrated. ${ }^{22,23}$ These nanostructures are promising candidates for light-emitting devices for wavelengths 1.3 and $1.55 \mu \mathrm{m} .{ }^{24,25}$ They have rectangular shape and show a broad photoluminescence (PL) spectrum, with several peaks that correspond to different heights of the QWRs which can differ by a number of monolayers. ${ }^{24}$

The aim of the present paper is to investigate the magnetic-field dependence of the exciton energy in such QWRs. We consider V-shaped GaAs/ $\mathrm{Al}_{0.4} \mathrm{Ga}_{0.6} \mathrm{As}$ QWRs studied experimentally in Ref. 6 and self-assembled rectangular InAs/InP QWRs which were investigated experimentally in Refs. 24 and 25 in the presence of an externally applied magnetic field. In our approach we include the true geometrical shape of the wire, as well as the mass mismatch between the wire and the barrier. The calculations are based on a finite difference scheme within the single band effective mass approximation. Since strain is important for the formation of the self-assembled QWRs, we include the influence of strain on the confinement potential for both the electron and the hole. We calculate the diamagnetic shift of the exciton energy for both V-shaped and self-assembled QWRs when the magnetic field is along different directions of the QWR. We analyze different sizes and shapes of the QWRs and compare them with the experimental measurements. The effect of the Coulomb interaction between the electron and the hole is also studied.

The paper is organized as follows. In Sec. II, we describe briefly the method and the theoretical model. The influence of strain on the electronic structure in InAs/InP selfassembled QWRs is discussed in Sec. III. Section IV is devoted to the study of the Coulomb interaction in QWRs. In the last section, Sec. V, we present results of the excitonic spectrum in a magnetic field and make a detailed comparison with available experimental data.

\section{THEORY}

\section{A. Theoretical model and basic equations}

We deal with the optical properties near the fundamental band-gap energy for $\mathrm{GaAs} / \mathrm{Al}_{0.4} \mathrm{Ga}_{0.6} \mathrm{As}$ and $\mathrm{InAs} / \mathrm{InP}$ 


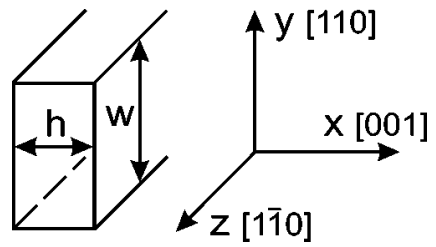

FIG. 1. Schematic illustration of a two-dimensional rectangular quantum box with height $h$ and width $w$ which reproduces the model of rectangular self-assembled InAs/InP QWR.

QWRs. We assume that the conduction and highest valence bands are decoupled. The full Hamiltonian for the system can be written in the form

$$
H=H_{e}+H_{h}+U\left(x_{e}-x_{h}, y_{e}-y_{h}, z_{e}-z_{h}\right),
$$

where $H_{e}$ is the electron Hamiltonian, $H_{h}$ is the hole Hamiltonian, and the Coulomb interaction between the electron and the hole is

$$
U(x, y, z)=-\frac{e^{2}}{\varepsilon \sqrt{x^{2}+y^{2}+z^{2}}},
$$

where $\varepsilon$ is taken as the average value of the wire and the barrier. We can assume this approximation, since there is only a small variation of $\varepsilon$ inside and outside the QWR.

Let us first introduce the Hamiltonian for the InAs/InP QWR. In this work, we consider the geometry of InAs/InP QWR as a two-dimensional (2D) rectangular quantum box with height $h$ along the $x$ direction and width $w$ along the $y$ direction. Different effective masses are assumed inside and outside the wire. We identify the crystal orientations [001], [110], and [1 $1 \overline{1} 0]$ with the $x, y$, and $z$ axes, as depicted in Fig. 1 , where $[1 \overline{1} 0]$ is the growth direction corresponding to the experiment ${ }^{24,25}$ situation.

The single-particle Hamiltonian for the electron in the presence of strain is the following:

$$
\begin{aligned}
H_{e}= & -\nabla_{x e} \frac{\hbar^{2}}{2 m_{e}^{*}(x, y)} \nabla_{x e}-\nabla_{y e} \frac{\hbar^{2}}{2 m_{e}^{*}(x, y)} \nabla_{y e}+E_{c e}(x, y) \\
& +a_{c} \varepsilon_{h y d}(x, y)
\end{aligned}
$$

where $m_{e}^{*}$ is the effective mass of the electron, $E_{c e}(x, y)$ is the unstrained conduction band offset, $a_{c}$ is the hydrostatic deformation potential for the conduction band, and $\varepsilon_{h y d}(x, y)$ denotes the hydrostatic strain. The Hamiltonian describing the heavy $(h h)$ and the light $(l h)$ hole states is, respectively, given by

$H_{h h}=-\nabla_{x h h} \frac{\hbar^{2}}{2 m_{h h}^{*}(x, y)} \nabla_{x h h}-\nabla_{y h h} \frac{\hbar^{2}}{2 m_{h h}^{*}(x, y)} \nabla_{y h h}+V_{v h h}(x, y)$,

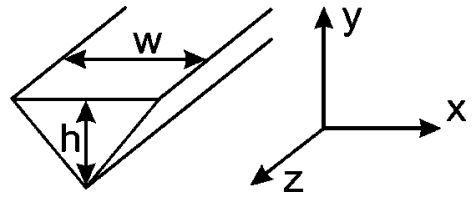

FIG. 2. Schematic illustration of a triangular-shaped QWR with height $h$ and base length $w$.

$$
H_{l h}=-\nabla_{x l h} \frac{\hbar^{2}}{2 m_{l h}^{*}(x, y)} \nabla_{x l h}-\nabla_{y l h} \frac{\hbar^{2}}{2 m_{l h}^{*}(x, y)} \nabla_{y l h}+V_{v l h}(x, y),
$$

where $m_{h h}^{*}, m_{l h}^{*}$ are the effective masses of the heavy and the light holes, respectively; $V_{v h h}(x, y)$ and $V_{v l h}(x, y)$ are the confinement potentials for both holes due to the band offsets and strain. To derive these potentials we use the diagonalized 4 $\times 4$ Pikus-Bir Hamiltonian ${ }^{26}$

$$
V_{v h h}(x, y)=E_{v h}(x, y)+P+\operatorname{sgn}(Q) \sqrt{Q^{2}+R R^{\dagger}+S S^{\dagger}},
$$

$$
V_{v l h}(x, y)=E_{v h}(x, y)+P-\operatorname{sgn}(Q) \sqrt{Q^{2}+R R^{\dagger}+S S^{\dagger}},
$$

where $E_{v h}(x, y)$ is the unstrained valence band offset between the wire and the barrier material, $P, Q, R$, and $S$ are matrix elements which can be calculated using the deformation potentials $a_{v}, b$, and $d$ of the valence band and the components of the strain tensor $\varepsilon$

$$
\begin{gathered}
P=a_{v}\left(\varepsilon_{x x}+\varepsilon_{y y}+\varepsilon_{z z}\right), \\
Q=\frac{b}{2}\left(\varepsilon_{x x}+\varepsilon_{y y}-2 \varepsilon_{z z}\right), \\
R=-\frac{\sqrt{3}}{2} b\left(\varepsilon_{x x}-\varepsilon_{y y}\right)+i d \varepsilon_{x y}, \\
S=-d\left(\varepsilon_{z x}-i \varepsilon_{y z}\right) .
\end{gathered}
$$

In order to obtain the strain components of the QWR within the square cross section, we adapted the method given by Downes. ${ }^{27} \varepsilon_{x x}$ and $\varepsilon_{y y}$ are determined as a function of the height and the width of the wire, while $\varepsilon_{z z}$ is equal to the misfit strain $\epsilon_{0}=\left(a_{0 \operatorname{InAs}}-a_{0 \operatorname{In} P}\right) / a_{0 \operatorname{In} P}$ within the strained QWR and equal to zero in the barrier. Therefore, the expression $\varepsilon_{h y d}(x, y)=\varepsilon_{x x}+\varepsilon_{y y}+\varepsilon_{z z}$ in the case of hydrostatic strain for the electron depends only on the $x$ - and $y$ coordinates, as well as the confinement potentials for the holes $V_{v h h}(x, y)$ and $V_{v l h}(x, y)$.

The Hamiltonian of the V-shaped wire for the electron and for the hole is the same as for the self-assembled wires, but without the strain parts. As in the previous case, we take different masses inside the wire and in the barrier. As shown in Fig. 2, we use for the simulation a triangular shape of the QWR with height $h$ and base length $w$.

In the case of an applied magnetic field the kinetic operator for the particles is given by 


$$
T^{e, h}(x, y)=\left(\mathbf{p}_{e, h}-\frac{q}{c} \mathbf{A}\right) \frac{1}{2 m_{e, h}^{*}}\left(\mathbf{p}_{e, h}-\frac{q}{c} \mathbf{A}\right),
$$

where $q$ is the charge of the particle, $\mathbf{p}=-i \hbar \nabla$ is the momentum, and $\mathbf{A}$ is the vector potential of the magnetic field. The gauge is chosen such that it simplifies the problem as much as possible.

When the magnetic field is applied along the wire growth direction, i.e., $\mathbf{B}=B \hat{z}$, we chose the symmetric gauge $\mathbf{A}$ $=(B / 2)(-y \hat{x}+x \hat{y})$. Then the aforementioned kinetic-energy term for the electron and the hole (the subscripts are left out of the equation) can be written as

$$
\begin{aligned}
T(x, y)= & p_{x} \frac{1}{2 m^{*}(x, y)} p_{x}+p_{y} \frac{1}{2 m^{*}(x, y)} p_{y}+\frac{q B y}{4 m^{*}(x, y) c} p_{x} \\
& +\frac{q B y}{4 c} \frac{p_{x}}{m^{*}(x, y)}-\frac{q B x}{4 m^{*}(x, y) c} p_{y}-\frac{q B x}{4 c} \frac{p_{y}}{m^{*}(x, y)} \\
& +\frac{q^{2} B^{2}}{8 c^{2} m^{*}(x, y)}\left(x^{2}+y^{2}\right) .
\end{aligned}
$$

The total diamagnetic contribution to the kinetic part consists of five terms, of which the first four are linear and the last one is an extra parabolic confinement. Note that the resulting problem becomes two-dimensional.

For the magnetic field applied parallel to the $y$ direction $\mathbf{B}=B \hat{y}$, the following nonsymmetric gauge is used: $\mathbf{A}=$ $-B x \hat{z}$. With this gauge the kinetic-energy term is

$$
\begin{aligned}
T(x, y)= & p_{x} \frac{1}{2 m^{*}(x, y)} p_{x}+p_{y} \frac{1}{2 m^{*}(x, y)} p_{y}+p_{z} \frac{1}{2 m^{*}(x, y)} p_{z} \\
& -\frac{q B x p_{z}}{c m^{*}(x, y)}+\frac{q^{2} B^{2} x^{2}}{2 c^{2} m^{*}(x, y)} .
\end{aligned}
$$

The total Hamiltonian (with the confinement) still commutes with $p_{z}$, and therefore the wave function in the $z$ direction can still be taken as a plane wave. The ground state is obtained for $p_{z}=0$.

Consider now the case of a magnetic field oriented along the $x$ direction. If we choose the vector potential as $\mathbf{A}=B y \hat{z}$, then the kinetic part of the Hamiltonian is given by the equation

$$
\begin{aligned}
T(x, y)= & p_{x} \frac{1}{2 m^{*}(x, y)} p_{x}+p_{y} \frac{1}{2 m^{*}(x, y)} p_{y}+p_{z} \frac{1}{2 m^{*}(x, y)} p_{z} \\
& +\frac{q B y p_{z}}{c m^{*}(x, y)}+\frac{q^{2} B^{2} y^{2}}{2 c^{2} m^{*}(x, y)} .
\end{aligned}
$$

Again, $p_{z}$ commutes with the Hamiltonian and as a result the problem is still $2 \mathrm{D}$.

\section{B. Solution methods}

The electron and hole states in the QWR were solved separately and can be described by the following Schrödinger equations:

$$
\begin{aligned}
H_{e} \Psi_{e}\left(x_{e}, y_{e}\right) & =E_{e} \Psi_{e}\left(x_{e}, y_{e}\right), \\
H_{h h} \Psi_{h h}\left(x_{h h}, y_{h h}\right) & =E_{h h} \Psi_{h h}\left(x_{h h}, y_{h h}\right),
\end{aligned}
$$

TABLE I. Input material parameters for InAs/InP and $\mathrm{GaAs} / \mathrm{Al}_{0.4} \mathrm{Ga}_{0.6} \mathrm{As} \mathrm{QWRs}$ used in the calculations: lattice constant $a_{0}$, band gap $E_{g}$ mass electron $m_{e}$ (Refs. 28-30), heavy hole mass $m_{h h}$, light hole mass $m_{l h}$, the hydrostatic deformation potential for the conduction band $a_{c}$, the deformation potentials of the valence band $a_{v}, b$, and $d$, strain coefficients $C_{11}$ and $C_{12}$, and dielectric constant $\varepsilon$.

\begin{tabular}{lcccc}
\hline \hline Parameter & InAs & InP & GaAs & $\mathrm{Al}_{0.4} \mathrm{Ga}_{0.6} \mathrm{As}$ \\
\hline$a_{0}(\AA)$ & 6.058 & 5.869 & --- & --- \\
$E_{g}(\mathrm{eV})$ & 0.417 & 1.424 & 1.519 & 2.018 \\
$m_{e}\left(m_{0}\right)$ & 0.023 & 0.077 & 0.067 & 0.1 \\
$m_{h h}[110]\left(m_{0}\right)$ & 0.513 & 0.885 & 0.51 & 0.61 \\
$m_{h h}(001)\left(m_{0}\right)$ & 0.333 & 0.532 & 0.51 & 0.61 \\
$m_{l h}\left(m_{0}\right)$ & 0.026 & 0.111 & --- & --- \\
$a_{c}(\mathrm{eV})$ & -5.08 & --- & --- & --- \\
$a_{v}(\mathrm{eV})$ & 1 & --- & --- & --- \\
$b(\mathrm{eV})$ & -1.8 & --- & --- & --- \\
$d(\mathrm{eV})$ & -3.6 & --- & --- & --- \\
$C_{11}(\mathrm{GPa})$ & 83.29 & --- & --- & --- \\
$C_{12}(\mathrm{GPa})$ & 45.26 & --- & --- & --- \\
$\varepsilon$ & 15.15 & 12.5 & 12.9 & 11.76 \\
\hline \hline
\end{tabular}

$$
H_{l h} \Psi_{l h}\left(x_{l h}, y_{l h}\right)=E_{l h} \Psi_{l h}\left(x_{l h}, y_{l h}\right)
$$

In order to solve these single-particle equations we used two independent numerical procedures; one is based on a twodimensional finite difference technique with uniform grid space, while the other relies on the finite element technique. In these methods the real 2D confinement potential and the different masses inside and outside of the QWR for the different particles were included. The values of the parameters used in the calculations are listed in Table I. All parameters have been taken from Ref. 31, unless indicated otherwise.

\section{Coulomb interaction}

In the next step, we consider the Coulomb interaction between the particles. Electron and hole are confined in the $x$ - and $y$ directions and can move freely along the wire direction. Because of the strong confinement in the $x y$ plane we are allowed to separate the $z$ motion from the lateral motion in the $x y$ plane and to assume that the Coulomb interaction has no effect on the $x y$ motion of the particles. In this case an approximate solution to Eq. (1) has the form

$$
\Psi\left(x_{e}, x_{h}, y_{e}, y_{h}, z\right)=\Psi_{e}\left(x_{e}, y_{e}\right) \Psi_{h}\left(x_{h}, y_{h}\right) \varphi(z),
$$

where $\varphi(z)$ is the wave function which describes the relative motion of the exciton in the $z$ direction, i.e. $z=z_{e}-z_{h}$. Averaging the Hamiltonian over the $x-y$ direction with the above wave functions, we obtain an effective one-dimensional equation for the motion in the wire direction 


$$
\left[E_{e}+E_{h}-\frac{\hbar^{2}}{2 \mu_{z}} \nabla_{z}^{2}+U_{e f f}(z)\right] \varphi(z)=E_{e x} \varphi(z),
$$

where $\mu_{z}$ is the reduced mass of the electron and hole along the wire axis and $U_{\text {eff }}(z)$ is the effective potential, which can be described by

$$
\begin{aligned}
& U_{e f f}(z)= \iiint \int d x_{e} d y_{e} d x_{h} d y_{h}\left|\Psi_{e}\left(x_{e}, y_{e}\right)\right|^{2} \mid \Psi_{h}\left(x_{h}, y_{h}\right) \\
& \times\left.\right|^{2} U\left(x_{e}-x_{h}, y_{e}-y_{h}, z\right) \\
& \frac{1}{\mu_{z}}= \iiint \int d x_{e} d y_{e} d x_{h} d y_{h}\left|\Psi_{e}\left(x_{e}, y_{e}\right)\right|^{2} \mid \Psi_{h}\left(x_{h}, y_{h}\right) \\
& \times\left.\right|^{2}\left(\frac{1}{m_{e}^{*}(x, y)}+\frac{1}{m_{h}^{*}(x, y)}\right) .
\end{aligned}
$$

In order to solve Eq. (13) we have to perform a fourdimensional integration to calculate the effective potential. We used two independent methods to calculate $U_{\text {eff }}(z)$ : a direct integration and an analytical method based on the Fourier transformation of the Coulomb potential. The exciton binding energy is then obtained from a solution of Eq. (13), which was solved numerically using a finite difference scheme.

\section{SELF-ASSEMBLED QUANTUM WIRES WITH STRAIN}

The formation of self-assembled InAs/InP QWRs is based on the strain-relaxation effect. It is therefore interesting and important to investigate the influence of strain on the electronic properties of the QWR. For the electron, as was defined in Eq. (3), the edge of the conduction band is shifted down by the hydrostatic strain $a_{c} \varepsilon_{\text {hyd }}$, which is $144 \mathrm{MeV}$ for InAs/InP QWR. It should be noted that in our strain calculation model this value is independent of the size of the QWR, because the sum of the normal strain components $\varepsilon_{\text {hyd }}=\varepsilon_{x x}+\varepsilon_{y y}+\varepsilon_{z z}$ is constant. ${ }^{32}$ This means that in the presence of strain the bottom of the electron confinement potential is shifted by the value of the hydrostatic strain.

In the case of the holes the situation is more complicated. The heavy and light hole band edges can be obtained from the aforementioned Pikus-Bir Hamiltonian, characterized in Sec. II by the formulas (5a) and (5b). In Fig. 3 the results for the profiles of the confinement potential for the heavy and light hole along the $x$ - and $y$ direction are compared. We plotted the case when the off-diagonal $R$ and $S$ matrices are neglected (dashed curves) and the case when the strain Hamiltonian includes all matrix elements (full curves). The first simple case gives the same behavior as for the conduction band, i.e., the edges of the valence band for the heavy and light holes are shifted by a value which depends on the $P$ and $Q$ diagonal matrix elements.

Further, we investigate the dimensional dependence of the heavy hole and light hole confinements for fixed width of the QWR as a function of the height, which is shown in Fig. 4. For this purpose we take the central point of the potentials and observe their dependence on the height of the QWR, as depicted by the full curves. Note that the heavy hole is above

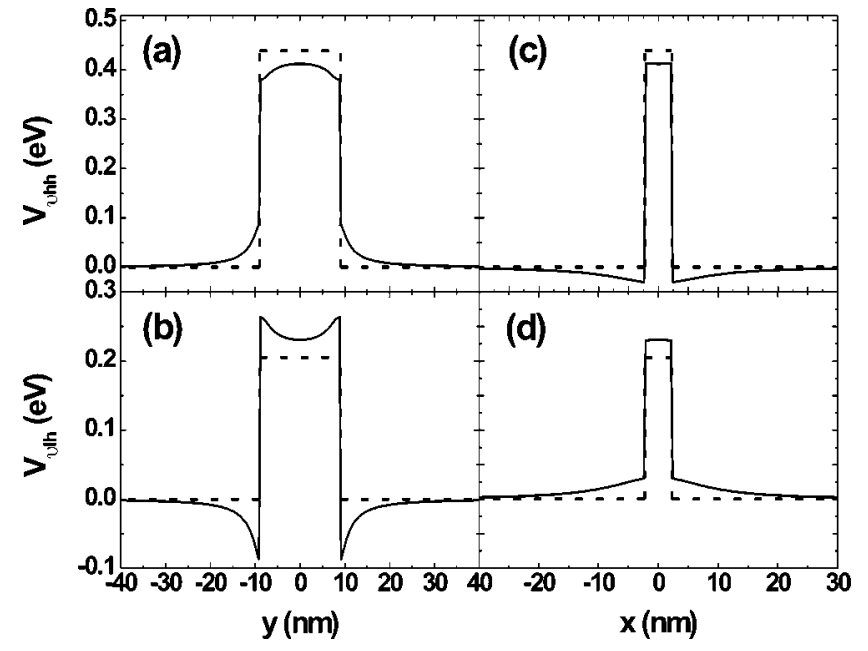

FIG. 3. Heavy hole [(a) and (c)] and light hole [(b) and (d)] band edges of InAs/InP QWR with $w=180 \AA$ and $h=15 \mathrm{ML}$ ( $1 \mathrm{ML}$ is $3.03 \AA$ ) along the $x$ - and $y$ direction. The full curves and dashed curves denote potentials calculated from the strain Hamiltonian with and without (shear potential) off-diagonal matrix elements, respectively.

the light hole states in the range of the height from 0 to $250 \AA$ and therefore is the ground state. This means that heavy and light holes in flat QWRs behave in a similar way as in quantum wells.

\section{CONTRIBUTION OF COULOMB INTERACTION TO THE EXCITON ENERGY}

Here, we investigate the Coulomb interaction between the electron and the hole in the wire. Using the wave functions from a numerical solution of the single-particle equation for the electron and the heavy hole, we perform a direct integration in order to calculate the effective Coulomb potential $U_{\text {eff }}(z)$ [see Eq. (14a)]. The heavy hole state is the ground state for the InAs/InP QWR, as was mentioned before, and for the GaAs $/ \mathrm{Al}_{0.4} \mathrm{Ga}_{0.6} \mathrm{As}$ QWRs, because the valence

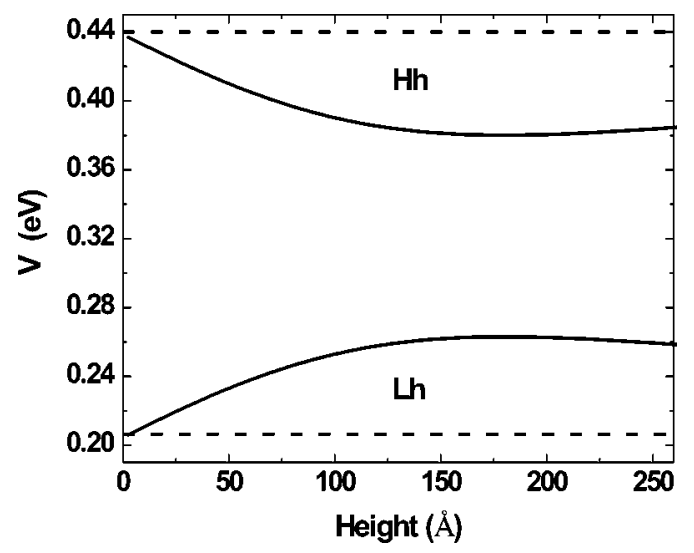

FIG. 4. The center of the confinement potentials for heavy hole and light hole as function of the height of the InAs/InP QWR with $w=180 \AA$. The dashed curves are the corresponding values for the shear potential. 


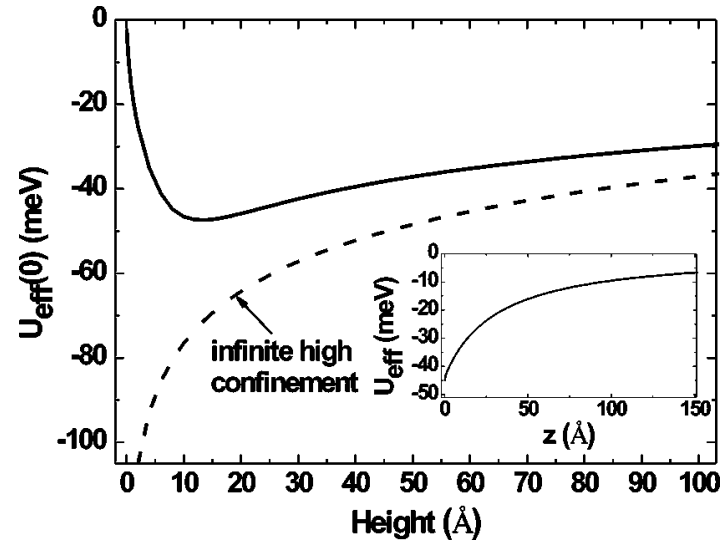

FIG. 5. The effective Coulomb potential at $z=0$ as a function of the height of the InAs/InP QWR with $w=180 \AA$. The inset shows the effective Coulomb potential as a function of the $z$ direction for a wire width $w=180 \AA$ and wire height $h=5 \mathrm{ML}$.

bound confinement depth is the same for the light and the heavy holes for these V-shape wires. In Fig. 5 we plot the effective potential $U_{\text {eff }}(0)$ as a function of InAs/InP wire height (solid curve) and compare the results with the case when the confinement potential along the $x$ - and $y$ direction is assumed to be infinitely high (dashed curve). The difference in the behavior for small values of the wire height is a result of the wave function spillover effect. ${ }^{33}$ For the infinity potential the wave function will always be confined inside the potential well, and the potential divergence at $z=0$ when $h \rightarrow 0$. The inset of Fig. 5 shows the calculated effective Coulomb potential for the InAs/InP QWR when $w=180 \AA$ and $h=5 \mathrm{ML}$.

In the next step, having the effective Coulomb potential, we solve the one-dimensional effective Schrödinger equation, Eq. (13), for the relative exciton wave function in the $z$ direction, in order to find the binding energy of the exciton. We used the finite difference technique. The wave function squared is shown in Fig. 6. We found that the binding energy of the exciton for the InAs/InP rectangular QWRs depends weakly on the height of the flat QWR. When the wire width is equal to $180 \AA$, which corresponds to the experimental

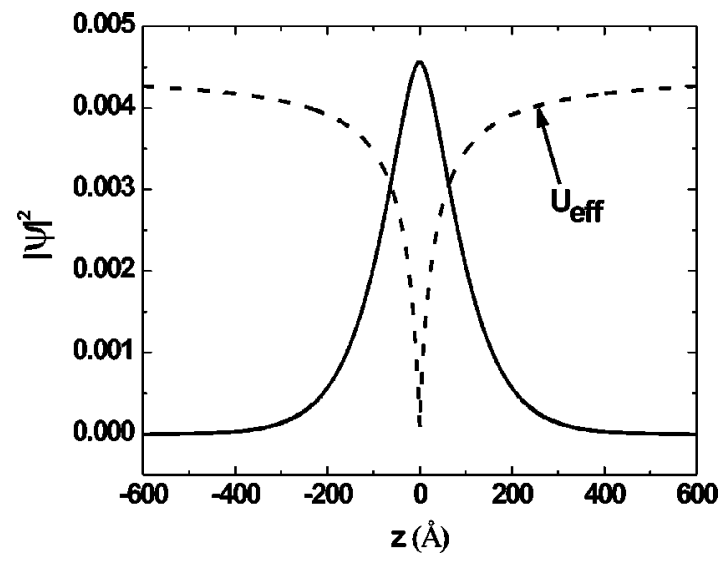

FIG. 6. The probability density (solid curve) and effective potential (dashed curve) of the exciton in InAs/InP QWR as a function of the $z$ direction for $w=180 \AA$ and $h=5 \mathrm{ML}$.

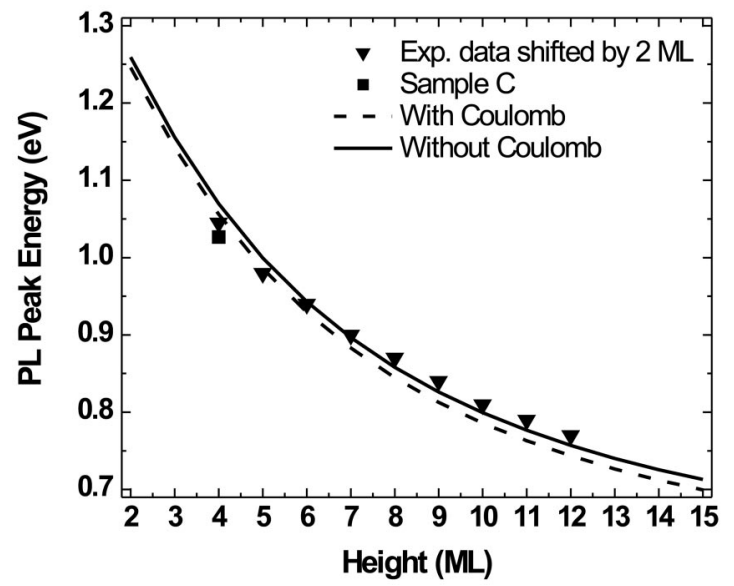

FIG. 7. PL peak energies as a function of the InAs/InP QWR height. The solid and dashed lines are the theoretical calculations for the heavy hole excitons with and without taking into account the Coulomb interaction, respectively. The triangles correspond to the experimental data from Ref. 24 shifted by $2 \mathrm{ML}$ and the square to sample $C$ from Ref. 33.

value, the binding energy of the electron-hole pair is 14.14 and $13.35 \mathrm{meV}$ for 5 and $10 \mathrm{ML}$, respectively. In the case of the V-shaped GaAs/ $/ \mathrm{Al}_{0.4} \mathrm{Ga}_{0.6} \mathrm{As}$ QWR the difference is more pronounced. When the wire height is $10 \mathrm{~nm}$ and base length 20 and $40 \mathrm{~nm}$, the Coulomb interaction between the electron and the heavy hole is 18.73 and $15.58 \mathrm{meV}$, respectively.

In Ref. 24 results were reported of PL experiments from an array of self-assembled QWRs. The PL spectrum consists of several peaks that correspond to emission from QWR with different height, which can vary by a discrete number of monolayers. The relation between the wire heights and the individual peaks in the PL spectrum are unknown. But, we know that the height of the QWR can only vary by a discrete number of monolayers; it gives us the exact freedom to shift the experimental data. In Ref. 33, it was proposed to shift the experimental data by $1 \mathrm{ML}$ in comparison with the results presented in Ref. 24. However, the calculated peak energies also had to be shifted up by $39 \mathrm{meV}$ in order to obtain a good agreement. In the present calculations, using parameters taken from Table I, we also included the Coulomb interaction between the electron and the hole. The best agreement in the shape of the calculated curves in Fig. 7 with (solid curve) and without (dashed curve) inclusion of the Coulomb interaction is obtained when the experimental data are shifted by $2 \mathrm{ML}$ in comparison with the results which were presented in Ref. 24. The small disagreement can be explained for example by the size dependence of the band gap in InAs/InP QWR, ${ }^{34}$ or by the uncertainty on the band offsets. $^{31}$

\section{QUANTUM WIRE STATES IN A MAGNETIC FIELD}

In this section we calculate the diamagnetic shifts for different directions of the magnetic field and compare with experimental measurements. In Fig. 8 we show the shift of the 


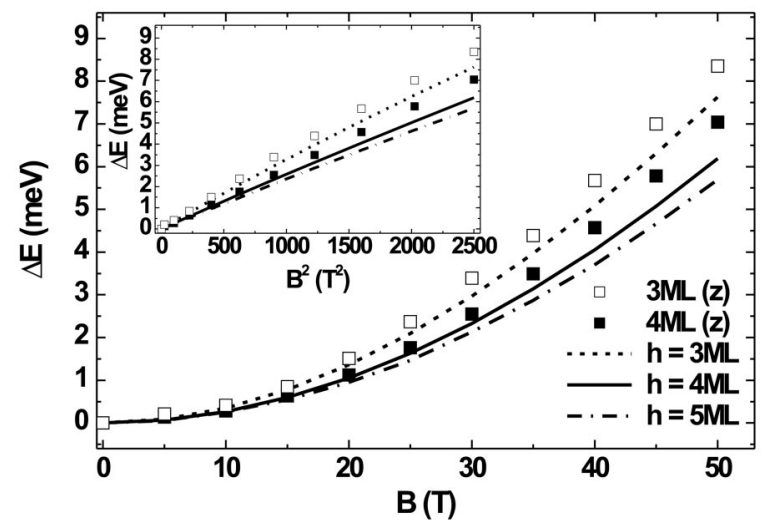

FIG. 8. The exciton diamagnetic shift as a function of magnetic field $(\mathbf{B} / / \hat{z})$ for the InAs/InP QWR. The dashed, full, and short dash-dot curves correspond to the numerical calculations done for different heights of the wire. The open squares and full squares represent the experimental data for the wire height 3 and $4 \mathrm{ML}$, respectively, with width equal to $180 \AA$. The inset shows the same dependencies using a quadratic scale for the magnetic field $B$.

PL peaks in the InAs/InP QWR for the magnetic field applied along the $z$ direction. We compare our theoretical results, which are denoted by three different curves, with the experimental data of Ref. 33 for QWRs with heights equal to 3 and $4 \mathrm{ML}$ and with width $180 \AA$. As the magnetic field increases, the wave functions are squeezed due to the extra confinement of the magnetic field. This squeezing leads to an increase in energy, as we see in Fig. 8. Up to $25 \mathrm{~T}$ a good agreement is obtained between the experimental points and the theoretical curves for the heights of 3 and $4 \mathrm{ML}$. The discrepancy between theory and experiment for both heights at $50 \mathrm{~T}$ is about $1 \mathrm{meV}$. The numerical results for the QWR with a height of $5 \mathrm{ML}$ are also shown for comparison.

When the magnetic length is much larger than the exciton diameter, the effect of the magnetic field can be treated as a perturbation and the resulting diamagnetic shift can be expressed as $\Delta E=\beta B^{2}$, with the diamagnetic coefficient $\beta$ $=e^{2}\left\langle\rho^{2}\right\rangle / 8 \mu$, where $\mu$ is the reduced mass of electron and hole, and $\langle\rho\rangle$ is the average quadratic distance between electron and hole. However, when the magnetic field is large enough, i.e., the magnetic length is comparable to the dimensions of the wire in the direction perpendicular to the applied field, the magnetic confinement becomes dominant and the parabolic dependence changes to a linear one, $\Delta E=\hbar \omega_{c}^{*}$, with $\omega_{c}^{*}=e B / m^{*}$. From the inset in Fig. 8 we see the fully parabolic behavior of the diamagnetic shift energy of the experimental data as a function of the magnetic field $B$. The theoretical curves show a very small deviation from a linear dependence.

Next, we investigated the effect of a magnetic field applied along the $y$ direction of the InAs/InP rectangular selfassembled QWR. Figure 9 shows the experimental results ${ }^{33}$ and the present theoretical calculations. Numerical results for three different heights of the wire are depicted. Again, a fair agreement between the experimental points and the theoretical curves is obtained. A more pronounced difference of $1.5 \mathrm{meV}$ for the height of $4 \mathrm{ML}$ at $50 \mathrm{~T}$ is observed in comparison with the previous figure, while for $3 \mathrm{ML}$ it is about

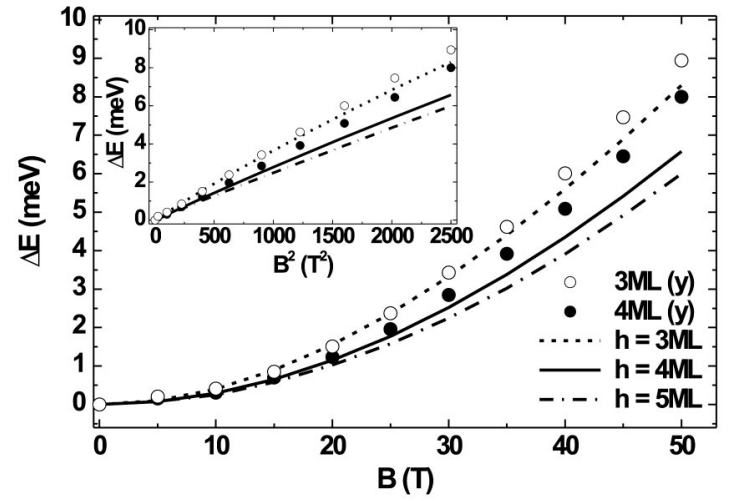

FIG. 9. The exciton diamagnetic shift as a function of magnetic field $(\mathbf{B} / / \hat{y})$ for the InAs/InP QWR. The dashed, full, and short dash-dot curves correspond to our numerical calculations for different heights of the wire. The open circles and full circles represent the experimental data for the wire height 3 and 4 ML, respectively, with width equal to $180 \AA$. The inset shows the same dependencies with a quadratic scale of the magnetic field $B$.

$1 \mathrm{meV}$. The deviation of the theoretical calculations from a linear dependence is seen for $B>25 \mathrm{~T}$ in the inset of Fig. 9 . The expected magnetic field value for which the magnetic length equals the height of $4 \mathrm{ML}$ is about $450 \mathrm{~T}$ (for $3 \mathrm{ML}$ it will be even larger), and thus much larger than $50 \mathrm{~T}$. But, because of the significant spread of the wave function into the barrier material of the QWR for such a narrow height we find already a deviation for smaller $B$ values. For instance, at the magnetic field $25 \mathrm{~T}$ the magnetic length is about $50 \AA$, which is the value of the wave function radii along the height direction. ${ }^{33}$

The reason for the small quantitative deviation between the experimental data and the theoretical curves at high magnetic field along the $z$-and the $y$ direction might be due to the following:

(1) We neglected the coupling between the heavy hole and the light hole which increases with increasing magnetic field. This causes only an insignificant deviation since the energy contribution of holes in the diamagnetic shift is very small in comparison with the contribution of the electron.

(2) The variation of $\varepsilon$ inside of the wire and in the barrier was not included. This can only slightly modify $(1-2 \mathrm{meV})$ the Coulomb term of the ground state for zero magnetic field for this rectangular QWR. However, the Coulomb energy between the electron and hole in InAs/InP QWR practically does not change for magnetic fields up to $50 \mathrm{~T}$, as we will see later. Therefore, the diamagnetic shift is not affected by including spatial dependence of $\varepsilon$.

(3) The most realistic reason for the discrepancy is that we assumed a rectangular shape for the QWR, which may not be the exact shape, and that we assumed an abrupt interface between the wire and the barrier material. But, because of the lack of detailed structural information of the QWR we are not able to do better without introducing extra unknown parameters.

Next, we present our results for the V-shaped GaAs/ $\mathrm{Al}_{0.4} \mathrm{Ga}_{0.6}$ As QWRs. The parameters used in the simulations are shown in Table I. Experimental results ${ }^{6}$ were re- 

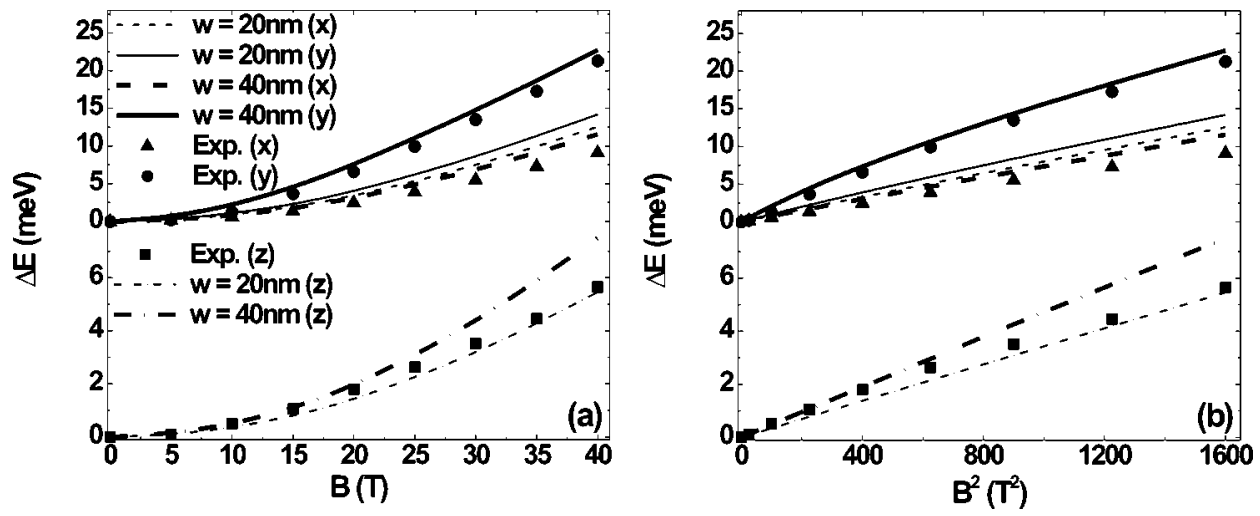

FIG. 10. The exciton diamagnetic shift as function of magnetic field $B$ for the $\mathrm{V}$-shaped $\mathrm{GaAs}_{2} / \mathrm{Al}_{0.4} \mathrm{Ga}_{0.6} \mathrm{As} \mathrm{QWRs}$ with $h=10 \mathrm{~nm}$ and for $w=20 \mathrm{~nm}$ (thin curves) and $w=40 \mathrm{~nm}$ (thick curves). The solid and dashed curves denote our numerical results for a magnetic field applied along the $y$ - and $x$ direction, whereas the dash-dot curves are for $\mathbf{B} / / \hat{z}$. The experimental data (Ref. 6) for the $x$-, $y$-, and $z$ direction of the magnetic field are indicated by full triangles, circles, and squares, respectively. The results are represented for a linear [(a)]and quadratic [(b)] of $B$ scale.

ported for vertically stacked triangular-shaped QWRs, of which the average base length $w$ and height $h$ were reported to be 20 and $10 \mathrm{~nm}$, respectively. The orientation of the magnetic field was applied along the $x-, y$-, and $z$ direction. In Fig. 10 the diamagnetic shift of the exciton ground-state energy is plotted as a function of the magnetic field for all three cases. For comparison, we show theoretical calculations for the QWR with a height of $10 \mathrm{~nm}$ and a base length of 20 and $40 \mathrm{~nm}$, according to Fig. 2. Note again that the diamagnetic shift reflects the lateral size of the wave function perpendicular to the magnetic field. Therefore, in Fig. 10(a) we can observe a rather large difference for the numerical curves with base length 20 and $40 \mathrm{~nm}$, when the magnetic field is applied in the $y$ direction, as well as in the $z$ direction (the base length is along the $x$ direction). And, as expected, when the magnetic field is applied along the $x$ direction, the variation is small; even at $40 \mathrm{~T}$ it is less than $1 \mathrm{meV}$. We also see that the qualitative agreement with the experimental measurements is best for $w=40 \mathrm{~nm}$ up to $20 \mathrm{~T}$, beyond which there are small deviations between theory and experiment which become substantial for $\mathbf{B} / / \hat{z}$. Figure 10(b) shows the magnetic dependencies of the $\mathrm{GaAs} / \mathrm{Al}_{0.4} \mathrm{Ga}_{0.6} \mathrm{As} \mathrm{QWR}$ as a function of $B^{2}$. The deviation from a linear behavior is found for both the numerical and the experimental curves. This is not surprising, since the lateral size of the wire is relatively large, and so the magnetic field becomes important even at small values.

We also investigate another shape of the V-shaped $\mathrm{GaAs} / \mathrm{Al}_{0.4} \mathrm{Ga}_{0.6} \mathrm{As}$ QWR, as suggested by the highresolution secondary electron image of the wire in Ref. 6. In Fig. 11 we compare our simulations for this new shape, shown in the inset of Fig. 11(b). However, only for the $z$ direction is a good fit with experimental measurements obtained, while for the other two directions of the magnetic field a large discrepancy between theory and experiment is found. Accordingly, it should be stressed that the shape of the wire confinement plays a rather important role in the simulation of the magnetic properties for QWRs. The best fit between the experimental points and the numerical curves is obtained for the shape mentioned in Fig. 2 with $h=8.6 \mathrm{~nm}$ and $w=37 \mathrm{~nm}$, as plotted in Fig. 12. As we can see from Figs. 12(a) and 12(b), full agreement is observed in the whole $B$ region, for each direction of magnetic field. In comparison with the experimentally determined dimensions of
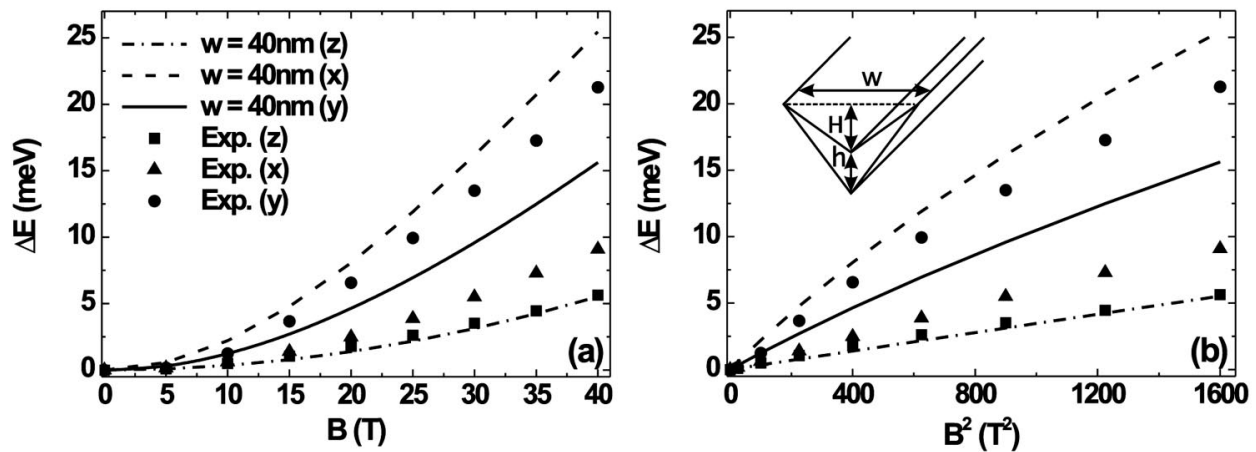

FIG. 11. The exciton diamagnetic shift as function of magnetic field $B$ for V-shaped $G a A s / \mathrm{Al}_{0.4} \mathrm{Ga}_{0.6} \mathrm{As}$ QWR with the profile shown in the inset (b). The heights of the QWR $h=10 \mathrm{~nm}$ and $H=19.3 \mathrm{~nm}$, the base length $w=40 \mathrm{~nm}$. The solid and dashed curves denote our numerical calculation for the magnetic field applied along the $y$ - and $x$ direction, whereas the dash-dot curves are for $\mathbf{B} / / \hat{z}$. The experimental data (Ref. 6) for the $x$-, $y$-, and $z$ direction of the magnetic field are indicated by full triangles, circles, and squares, respectively. 

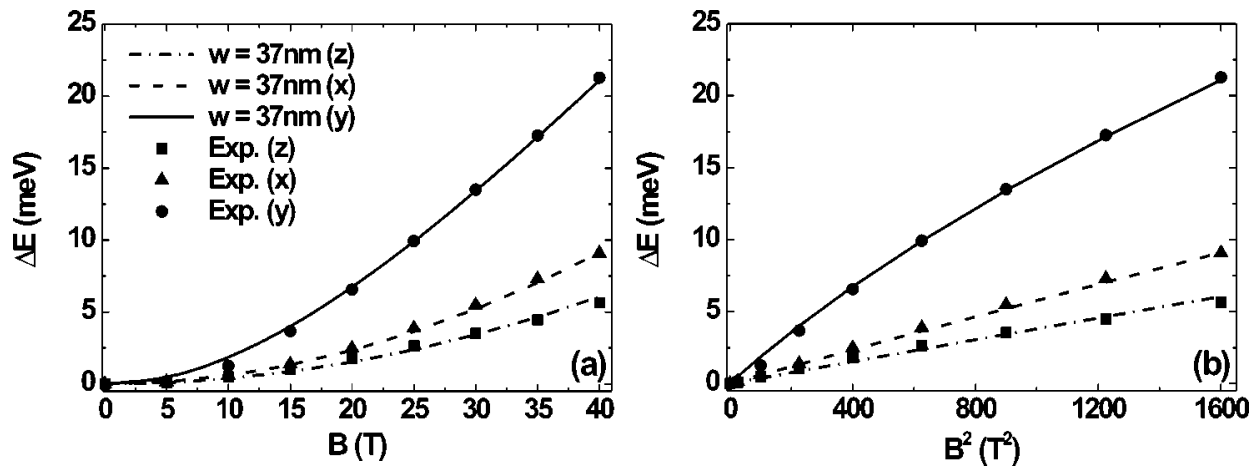

FIG. 12. The exciton diamagnetic shift as function of magnetic field $B$, for the V-shaped $\mathrm{GaAs} / \mathrm{Al}_{0.4} \mathrm{Ga}_{0.6} \mathrm{As} \mathrm{QWR}$ with $h=8.6 \mathrm{~nm}$ and $w=37 \mathrm{~nm}$. The solid and dashed curves denote numerical calculation for the magnetic field applied along the $y$ - and $x$ direction, whereas the dash-dot curves are for $\mathbf{B} / / \hat{z}$. The experimental data (Ref. 6) for the $x-, y-$, and $z$ direction of the magnetic field are indicated by full triangles, circles, and squares, respectively.

the QWR, the height of $8.6 \mathrm{~nm}$ is about the same as the aforementioned experimental average height of $10 \mathrm{~nm}$; however, the base length $w=37 \mathrm{~nm}$ is found to be almost twice as large, as mentioned in the experimental paper, ${ }^{6}$ namely $20 \mathrm{~nm}$. However, from the high-resolution electron image of the wire in Ref. 6, the value of $20 \mathrm{~nm}$ as base length seems indeed to be a clear underestimation.

Next, we discuss the influence of the magnetic field on the exciton binding energy. In Fig. 13 we show the density for the electron and the heavy hole for both InAs/InP and V-shaped GaAs $/ \mathrm{Al}_{0.4} \mathrm{Ga}_{0.6}$ As QWRs at different values of the magnetic field. Note that for both cases the effect of the applied field enhances the particle confinement into the wire, particularly for the wider V-shaped wires. This is also confirmed when comparing the Coulomb interaction. For the InAs/InP QWR the Coulomb contribution to the exciton energy is 13.79 and $13.89 \mathrm{meV}$ for 0 and $50 \mathrm{~T}$, respectively, while for $\mathrm{GaAs} / \mathrm{Al}_{0.4} \mathrm{Ga}_{0.6} \mathrm{As}$ the difference is larger: 16.72 and $18.93 \mathrm{meV}$ for 0 and $40 \mathrm{~T}$, respectively.

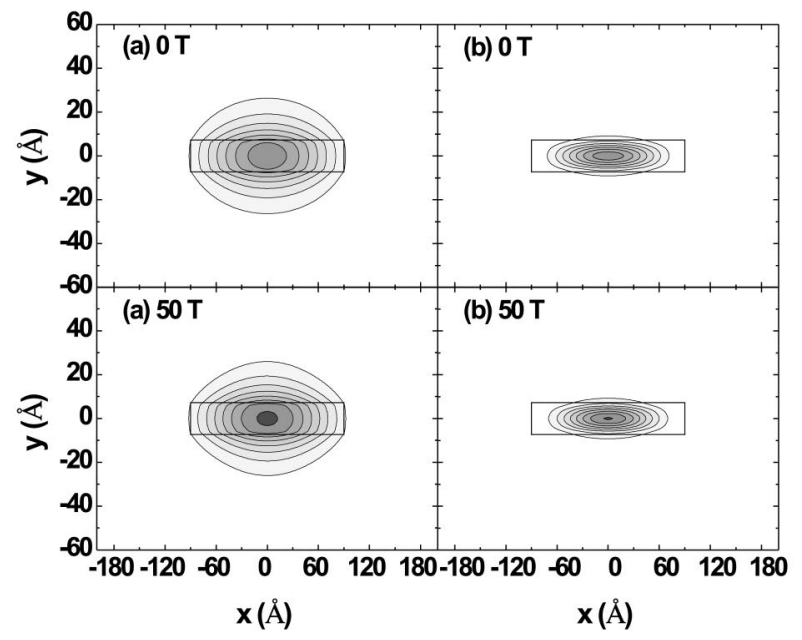

\section{CONCLUSIONS}

In summary, the excitonic properties in the presence of a magnetic field in InAs/InP self-assembled and V-shaped GaAs $/ \mathrm{Al}_{0.4} \mathrm{Ga}_{0.6} \mathrm{As}$ QWRs were investigated theoretically. We include the effect of strain on the electronic structure of InAs/InP QWRs. The heavy hole states are found to be the ground states for both types of wires. We show that the Coulomb interaction energy between the electron-hole pair has a relatively small value for the considered self-assembled QWRs and a very small change is observed, as the wire height increases. For the V-shaped wire the Coulomb energy between the particles depends more strongly on the dimensions of the wire.

By calculating the effective potential in the rectangular InAs/InP QWR, we confirm the spillover effect in the case of flat wires with a height much narrowed as compared to its width. We also compare our theoretical results with the experimental measurements on the PL peak energies in the selfassembled QWR. A good agreement is reached between

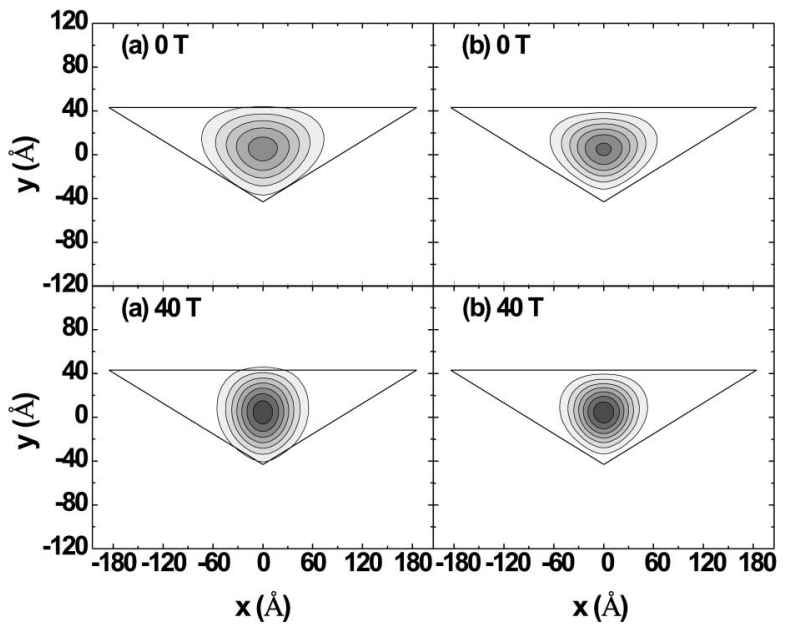

FIG. 13. The contour plot of the densities in the rectangular InAs/InP (left figure) and the V-shape $\mathrm{GaAs} / \mathrm{Al}_{0.4} \mathrm{Ga}_{0.6} \mathrm{As}$ (right figure) QWRs for electron [(a)] and heavy hole [(b)] at magnetic field 0, 40, and $50 \mathrm{~T}$. 
them in the region of the height of the wire from 4 to $13 \mathrm{ML}$, when the width is fixed and taken equal to $180 \AA$.

Numerical simulations of the diamagnetic shift are performed for InAs/InP self-assembled QWR. Deviation from the experiment at $B=50 \mathrm{~T}$ is found to be less than $1.5 \mathrm{meV}$ when the magnetic field is applied along the $y$ direction. For the $z$ direction of the magnetic field it is even smaller than $1 \mathrm{meV}$. Calculations for three difference heights of the wire are compared. Further, the results for the diamagnetic shift for the $\mathrm{GaAs} / \mathrm{Al}_{0.4} \mathrm{Ga}_{0.6} \mathrm{As} \mathrm{QWR}$ show a very good agreement between the experimental data and the theoretical curves, when the shape of the wire is considered as triangu- lar. The nonparabolic dependence as a function of $B$ starts at a small magnetic field value for both the experimental and the theoretical data, which is a consequence of the larger dimensions of the V-shaped wire.

\section{ACKNOWLEDGMENTS}

This work was supported by the Flemish Science Foundation (FWO-Vl) and the European Commission Network of Excellence: SANDiE. A. Matulis, J. Davies, M. Hayne, and V. Moshchalkov are acknowledged for fruitful discussions.
*Electronic address: sidor.yosyp@ua.ac.be

†Electronic address: francois.peeters@ua.ac.be

${ }^{1}$ Y. Arakawa and H. Sakaki, Appl. Phys. Lett. 40, 939 (1982).

${ }^{2}$ H. Temkin, G. J. Dolan, M. B. Panish, and S. N. G. Chu, Appl. Phys. Lett. 50, 413 (1987).

${ }^{3}$ W. Wegscheider, L. N. Pfeiffer, M. M. Dignam, A. Pinczuk, K. W. West, S. L. McCall, and R. Hull, Phys. Rev. Lett. 71, 4071 (1993).

${ }^{4}$ H. Akiyama, T. Someya, M. Yoshita, T. Sasaki, and H. Sakaki, Phys. Rev. B 57, 3765 (1998).

${ }^{5}$ H. Akiyama, and M. Yoshita, L. N. Pfeiffer, K. W. West, and A. Pinczuk, Appl. Phys. Lett. 82, 379 (2003)

${ }^{6}$ Y. Nagamune, Y. Arakawa, S. Tsukamoto, M. Nishioka, S. Sasaki, and N. Miura, Phys. Rev. Lett. 69, 2963 (1992).

${ }^{7}$ T. Arakawa, Y. Kato, F. Sogawa, and Y. Arakawa, Appl. Phys. Lett. 70, 646 (1997).

${ }^{8}$ G. Goldoni, F. Rossi, E. Molinari, A. Fasolino, R. Rinaldi, and R. Cingolani, Appl. Phys. Lett. 69, 2965 (1996).

${ }^{9}$ F. Rossi and E. Molinari, Phys. Rev. Lett. 76, 3642 (1996).

${ }^{10}$ K. Chang and J. B. Xia, Phys. Rev. B 58, 2031 (1998).

${ }^{11}$ M. H. Degani and O. Hipólito, Phys. Rev. B 35, R9345 (1987).

${ }^{12}$ F. Rossi and E. Molinari, Phys. Rev. B 53, 16462 (1996).

${ }^{13}$ S. Glutsch, and F. Bechstedt, W. Wegscheider, and G. Schedelbeck, Phys. Rev. B 56, 4108 (1997).

${ }^{14}$ S. N. Walck, T. L. Reinecke, and P. A. Knipp, Phys. Rev. B 56, 9235 (1997).

${ }^{15}$ K. Chang and J. B. Xia, Phys. Rev. B 58, 2031 (1998).

${ }^{16}$ G. Goldoni, F. Rossi, E. Molinari, and A. Fasolino, Phys. Rev. B 55, 7110 (1997).

${ }^{17}$ O. Stier and D. Bimberg, Phys. Rev. B 55, 7726 (1997).

${ }^{18}$ T. Sogawa, H. Ando, S. Ando, and H. Kanbe, Phys. Rev. B 56, 1958 (1997).
${ }^{19}$ F. V. Kyrychenko and J. Kossut, Phys. Rev. B 61, 4449 (2000).

${ }^{20}$ G. W. Bryant and Y. B. Band, Phys. Rev. B 63, 115304 (2001).

${ }^{21}$ J. R. Madureira, M. Z. Maialle, and M. H. Degani, Phys. Rev. B 66, 075332 (2002).

${ }^{22}$ L. González, J. M. García, R. García, J. Martínez-Pastor, C. Ballesteros, and F. Briones, Appl. Phys. Lett. 76, 1104 (2000).

${ }^{23}$ H. R. Gutiérrez, M. A. Gotta, and M. M. G. de Carvalho, Appl. Phys. Lett. 79, 3854 (2001).

${ }^{24}$ B. Alén, J. Martínez-Pastor, A. García-Cristobal, L. González, and J. M. García, Appl. Phys. Lett. 78, 4025 (2001).

${ }^{25}$ B. Alén, J. Martínez-Pastor, L. González, J. M. García, S. I. Molina, A. Ponce, and R. García, Phys. Rev. B 65, 241301 (R) (2002).

${ }^{26}$ Calvin Yi-Ping Chao and Shun Lien Chuang, Phys. Rev. B 46, 4110 (1992).

${ }^{27}$ J. Downes and D. A. Faux, J. Appl. Phys. 77, 2444 (1995).

${ }^{28}$ M. Grundmann, O. Stier, and D. Bimberg, Phys. Rev. B 52, 11969 (1995).

${ }^{29}$ Jasprit Singh, Semiconductor Devices: Basic Principles (Wiley, New York, 2001).

${ }^{30}$ F. B. Pedersen and Yia-Chung Chang, Phys. Rev. B 55, 4580 (1997).

${ }^{31}$ I. Vurgaftman, J. R. Meyer, and L. R. Ram-Mohan, J. Appl. Phys. 89, 5815 (2001).

${ }^{32}$ D. A. Faux, J. R. Downes, and E. P. O'Reilly, J. Appl. Phys. 80, 2515 (1996).

${ }^{33}$ J. Maes, M. Hayne, Y. Sidor, B. Partoens, F. M. Peeters, Y. González, L. González, D. Fuster, J. M. García, and V. V. Moshchalkov, Phys. Rev. B 70, 155311 (2004).

${ }^{34}$ H. Yu, J. Li, R. A. Loomis, Lin-Wang Wang, and W. E. Buhro, Nat. Mater. 2, 517 (2003). 